\title{
Masking of Antioxidant Capacity by the Interaction of Pancreatic $\alpha$-Amylase and Peanut Shells Polyphenols
}

\author{
Feng Gao, Yujiang, Cheng, Yali Yu ${ }^{\text {a }}$, Lvsha Gao \\ Department of Food Science and Engineering, Jilin University, Changchun, 130062, China \\ ${ }^{a}$ E-mail: yuyal@jlu.edu.cn, ${ }^{a}$ corresponding author
}

Keywords: Peanut Shells Polyphenols; Pancreatic $\alpha$-Amylase; Interactions; Antioxidant Capacity

\begin{abstract}
The antioxidant capacity of peanut shell polyphenols interacted with pancreatic $\alpha$-amylase is examined in vitro. The results indicated that peanut shells polyphenols could be used as a kind of potential natural antioxidant for food. The polyphenols easier binding to proteins made its scavenging activities decreased in complex biological mixtures when binding to the $\alpha$-amylase which include phlorglucinol, luteolin, pyrogallol, and catechin. From the results, the decreasing extention had a tight relationship with the structure of polyphenol. A carbony group at C (4) in the polyphenolic molecule showed great effect on interaction between polyphenols and $\alpha$-amylase which leading a higher decrease in scavenging activities of peanut shells polyphenols. And meta-position polyphenolic hydroxyls were more active than adjacent polyphenolic hydroxyls in the interaction with $\alpha$-amylase.
\end{abstract}

\section{Introduction}

Peanut shells is a rich by-product of peanut (Arachis hypogea.L) in China. Researches showed that peanut shells had functional components, such as polyphenols, carotene, isosaponaretin, and luteolin. At the same time, because of its antioxidative property, it likely has potential application as a kind of natural antioxidant for food. As it is known that the effect of antioxidants is often executed in complex biological mixtures where various interactions may take place. For example, polyphenol-protein complexes could significantly influence their biological activities have gain our concentration significantly when polyphenols bind to protein whether the outcome is soluble or not. This kind of binding will definitely affect the bioavailability whether polyphenols or protein ${ }^{[1]}$. Polyphenols-proteins binding may have many kinds of influence in bioavailability depending on the method of intake and amount and the structure ${ }^{[2,3]}$. Researches have shown that the antioxidant capacity of polyphenols could be modified by the presence of proteins when the experiment carried out in vitro ${ }^{[4,5]}$. The $\alpha$-amylase is widely distributed in animal saliva, pancreas, which is acting on starch and cut $\alpha-1$, 4-bond indiscriminately into maltose, glucose, $\alpha-1$, 6-bond limit dextrin. In this paper, the research is related to the relationship of interaction between the polyphenols and $\alpha$-amylase in a simulated artificial gastrointestinal. So, porcine pancreatic $\alpha$-amylase was chosen which extracted from mammalian. Porcine pancreatic $\alpha$-amylase is more suitable for the experiment for it is more similar to the human enzyme because of porcine pancreatic $\alpha$-amylase and the $\alpha$-amylase in the human body are the same as the $\alpha$-amylase, its molecular weight is about $46 \mathrm{kD}$, the optimum $\mathrm{pH}$ is $6.7 \sim 7^{[6]}$.

Therefore, the antioxidant capacity of peanut shells polyphenols interacted with pancreatic $\alpha$-amylase in vitro is examined in this paper. The trolox equivalent antioxidant capacity (TEAC) assay is used to evaluate antioxidant capacity. In this assay the capacity of a compound to scavenge the stable ABTS radical gives the antioxidant capacity ${ }^{[7,8]}$. The TEAC is used for evaluating the antioxidant capacity of both single compounds as well as antioxidant mixtures. In the present study this interaction was studied by adding antioxidants to pancreatic $\alpha$-amylase in vitro and assessing the antioxidant capacity of the mixture. 


\section{Materials and Methods}

Peanut were purchased from a local market. The polyphenol samples employed for HPLC analysis include pyrogallol, catechol, quercetin, phlorglucinol, luteolin and ABTS were all purchased from Sigma Ltd (United States). Porcine pancreatic $\alpha$-Amylase was purchased from Amresco Company (United States).

Manufacturing process of peanut shells polyphenols extracts (PSPE). Peanut was decorticated and the crude shells were smashed into powder ( 80 mesh/inch, $0.177 \mathrm{~mm}$ ) by shredder for preparing the PSPE. A sample of peanut shells $(50.0 \mathrm{~g})$ was defatted with $n$-hexane $(3400.0 \mathrm{~mL}, 10 \mathrm{~h})$ at ambient temperature, and then $n$-hexane was removed from peanut shells powder under reduced pressure in a vacuum oven. The PSPE was produced as below: The defatted peanut shells (10.0 g) were macerated for 4 min with $250 \mathrm{~mL}$ aqueous ethanol (75 \%, v/v) under $300 \mathrm{MPa}$ by a high pressure equipment (DL700, Da-long High-pressure Equipment Plant, Shanghai, China). After filtered, the residue was extracted again under the same conditions. The combined filtrates were evaporated to a final volume of $10.0 \mathrm{~mL}$ in a rotary evaporator at $35^{\circ} \mathrm{C}$ under vacuum. The concentrated solution was lyophilised with a freeze dryer system to obtain the PSPE as a brown powder. Total polyphenols content of PSPE were determined by A Folin-Ciocalteu method. The total phenolic content of PSPE was expressed as a gallic acid equivalent (g gallic acid/g skin) with gallic acid used as standard.PSPE were a complex mixture, mostly made up of luteolin, pyrogallol, catechol, phlorglucinol, quercetin. The peaks of five representative fractions have been quantified comparing with standard sample (pyrogallol; catechol; phlorglucinol; quercetin; luteolin). The biggest amount in PSPE was luteolin and other ingredients followed up. The quantity of luteolin is about $76 \%$ of total PSPE. The profiles analyzed by HPLC were shown in Fig. ${ }^{[11]}$. The molecular structures of luteolin, pyrogallol, catechol, phlorglucinol, quercetin were shown in Fig.2

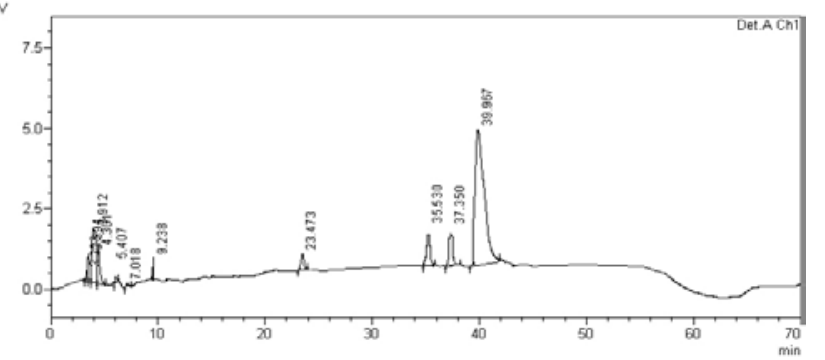

Fig.1 Profiles of PSPE by HPLC
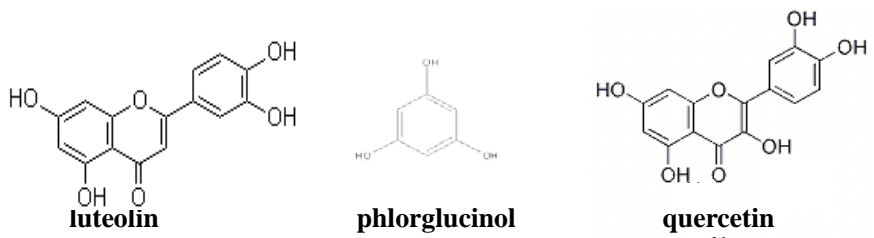

quercetin
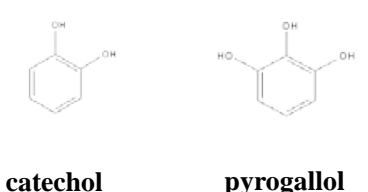

Fig.2 Molecular structures of luteolin, pyrogallol, catechol, phlorglucinol, quercetin

The TEAC assay measures the total radical scavenging capacity based on the ability of a compound to scavenge the stable ABTS radical (ABTS·). The absorbance of this radical was spectrophotometrically determined at $734 \mathrm{~nm}$ which produced through the reaction between ABTS and potassium persulfate. A $7 \mathrm{mM}$ solution of ABTS in milli-Q water (18.2 $\left.\mathrm{M} \Omega^{-\mathrm{cm}}\right)$ was prepared and ABTS. was formed after addition of potassium persulfate to the mixture in a final concentration of $2.45 \mathrm{mM}$. Under $37^{\circ} \mathrm{C}$, after a $12.0-16.0 \mathrm{~h}$ incubation at ambient temperature, the stock solution was diluted with PBS (pH7.4) untill an absorbance of the control of $0.70( \pm 0.02)$ at $734 \mathrm{~nm}$ was reached. The assay carried out as it has be described by $\mathrm{Re}^{[7]}$. It is given a decolorization by scavenging the radical when antioxidants were added to this radical solution. The degree of the decolorization indicates the antioxidants capacity. TEAC values were calculated by dividing the 
antioxidant-induced reduction in absorbance of the ABTS solution by the trolox-induced reduction in absorbance of the ABTS solution $(0.0257 \mathrm{~mm} / \mathrm{cm})$. A PSPE, pyrogallol, catechol, phlorglucinol, quercetin and luteolin were prepared in ethanol as stock solutions. At the same time, the BSA was dissolved in Milli-Q water. An antioxidant containing solution $(10.0 \mathrm{ml})$ and porcine pancreatic $\alpha$-Amylase solution $(10.0 \mu \mathrm{l})$ was added to PBS (final volume $500.0 \mu \mathrm{l}$ ) and incubated for $30.0 \mathrm{~min}$ at $37^{\circ} \mathrm{C}$. Then, $500.0 \mu \mathrm{l}$ ABTS s solution was added for determinating the antioxidant capacity. The absorbance was measured after mixing the compounds immediately. Therefore, the relative difference between the measured increase in antioxidant capacity is defined as the masking due to addition of an antioxidant compared to the calculated increase based on an additive effect at $t=30.0$ min. All experiments were repeated three times. All data were presented as mean $\pm \mathrm{SD}$ for three replicates for each sample. Statistical significance was accepted at a value of $p<0.05$.

\section{Results and discussion}

Interactions between polyphenols and proteins have been described. Current research demonstrates that the methanolic extract $(1 \mathrm{mg} / \mathrm{ml})$ of raw seed materials of P. pin-nata showed high inhibition of $\alpha$-amylase. However, little is known about the influence these interactions might have on the antioxidant capacity of polyphenols. In this experiment, PSPE were added to $\alpha$-amylase in vitro and the antioxidant capacity of the mixture was assessed to determine whether $\alpha$-amylase can affect the antioxidant capacity of polyphenols.

ABTS was oxidated by $\mathrm{K}_{2} \mathrm{~S}_{2} \mathrm{O}_{8}$ to ABTS+·, with the color of blue-green. Its absorbance was estimated and marked as $\mathrm{A}_{0}(734 \mathrm{~nm}) . \mathrm{A}_{\mathrm{i}}$ presents the absorbance on subsequent additions of polyphenol in the same ABTS+· solution. Scavenge ratio of different polyphenols to free radical ABTS+·, as in Eq. (1):

$$
\mathrm{S}_{\mathrm{Ri}}(\%)=\left[\left(\mathrm{A}_{0}-\mathrm{A}_{\mathrm{i}}\right) / \mathrm{A}_{0}\right] \times 100 \%
$$

Results obtained in the TEAC assay showed that polyphenol-rich extractions from peanut shells had the ability to scavenge free radical ABTS+· Activities (Fig.3). At the concentration of 20 and 1 $\mu \mathrm{g} / \mathrm{mL}$, its $\mathrm{S}_{\mathrm{R}}$ was $15.94 \%$ and $5.54 \%$, respectively. This indicated that the PSPE had obvious antioxidant capacity which was unanimous with previous study on the antioxidant activity of peanut shells polyphenols. Results also indicated that structures of polyphenols had a tent relationship with its ability of scavenging free radical ABTS $+\cdot$. Scavenge ratio of ABTS $+\cdot C_{i}(\%)$ for peanut shells polyphenols, catechin, phlorglucinol, luteolin and pyrogallol ranked in the following order: peanut shells polyphenols $>$ luteolin $>$ phlorglucinol $>$ pyrogallol $>$ catechin. This trend corresponds with the reducing number of phenolic hydroxyl in structure, except phlorglucinol and pyrogallol (Fig.3).

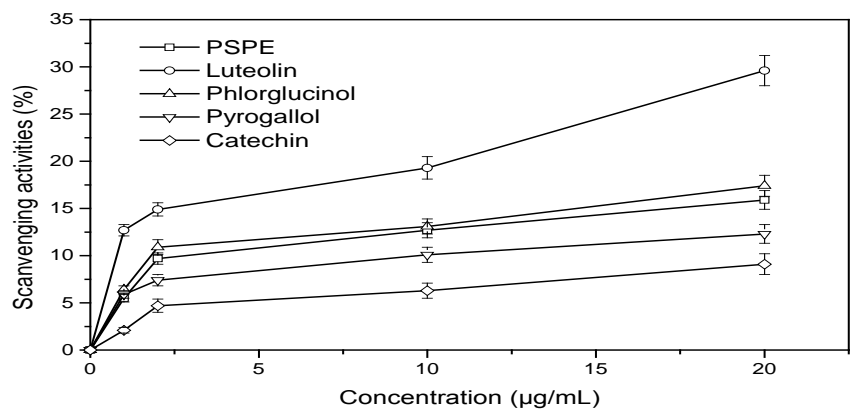

Fig.3 Scavenging free radical ABTS+· activities

Reported data show the more phenolic hydroxyl in polyphenol molecular, the stronger ability there was to scavenge free radical. In the case of phlorglucinol and pyrogallol, with the same number of polyphenolic hydroxyls, phlorglucinol showed higher free radical scavenger activity than pyrogallol (17.45\% and 12.3\%, respectively, at the concentration of $20 \mu \mathrm{g} / \mathrm{mL}$ ). Explanation may exist in the steric hindrance. Phlorglucinol has meta-polyphenolic hydroxyls while pyrogallol has adjacent polyphenolic hydroxyls. Part of polyphenolic hydroxyls ability was hidden because of their hindrance. 
Scavenging ratios of different peanut shells polyphenols to ABTS $+\cdot$ were tested again after polyphenols were incubated with $\alpha$-amylase at $37^{\circ} \mathrm{C}, 10$ minutes. Compared with former results, the reduction proportion (Rp) of polyphenols scavenging ratio for ABTS+· was counted as in Eq. (2):

$$
\mathrm{Rp}_{\mathrm{i}}(\%)=\left[\left(\mathrm{S}_{\mathrm{Ri}}-\mathrm{S}_{\mathrm{Ri}}{ }^{\prime}\right) / \mathrm{S}_{\mathrm{Ri}}\right] \times 100 \%
$$

$\mathrm{Rp}_{\mathrm{i}}$ : reduction proportion of polyphenols' scavenging ratio for ABTS+·;

$\mathrm{S}_{\mathrm{Ri}}$ : scavenge ratio of different polyphenols to free radical ABTS+·;

$\mathrm{S}_{\mathrm{Ri}}$ ': scavenge ratio of different polyphenols to free radical ABTS+·after reacting with $\alpha$-amylase.

Table 1 shows the Rp of different peanut shells polyphenols scavenging ratio for ABTS $+\cdot$ at the concentration of $20 \mu \mathrm{g} / \mathrm{mL}$ after being incubated with $\alpha$-amylase at $37^{\circ} \mathrm{C}$ for 10 minutes.

In table1, it showed that all the peanut shells polyphenols gave higher absorbance after incubated with $\alpha$-amylase. Accordingly, peanut shells polyphenols proved lower free radical scavenger activities when they interacted with $\alpha$-amylase (Fig.4).

Table1 Rp of different peanut shells polyphenols scavenging ratio

\begin{tabular}{lccccc}
\hline & $\begin{array}{c}\text { Peanut shells } \\
\text { polyphenls }\end{array}$ & luteolin & phlorglucinol & pyrogallol & catechin \\
\hline $\mathrm{S}_{\mathrm{R}} \%$ & $15.94 \pm 0.99$ & $29.44 \pm 1.60$ & $17.45 \pm 1.11$ & $12.30 \pm 0.99$ & $9.12 \pm 0.98$ \\
$\mathrm{~S}_{\mathrm{R}} \%$ & $10.87 \pm 0.99$ & $20.16 \pm 0.97$ & $14.43 \pm 0.98$ & $9.94 \pm 0.50$ & $7.74 \pm 0.60$ \\
$\mathrm{Rp} \%$ & $31.83 \pm 0.00$ & $31.52 \pm 0.39$ & $17.31 \pm 0.12$ & $19.19 \pm 0.50$ & $15.13 \pm 0.39$ \\
\hline
\end{tabular}

This phenomenon might because the interaction between polyphenols and $\alpha$-amylase occurs in the reaction system. Proline-rich proteins in salivary can form complexes with and precipitate dietary polyphenols, an as a result, they constitute the primary mammalian defense directed against ingested tannins. Though all the polyphenols presented binding interaction with $\alpha$-amylase, but the influence degree of $\alpha$-amylase to the polyphenols' scavenging activity existed difference. The Rp for peanut shells polyphenol, catechin, phlorglucinol, luteolin and pyrogallol at $20 \mu \mathrm{g} / \mathrm{mL}$ were $31.83 \%, 15.13 \%, 31.52 \%, 19.19 \%, 17.13 \%$, respectively. Obviously, peanut shells polyphenols gave the biggest binding with $\alpha$-amylase, and catechin implied the lowest ability to interact with $\alpha$-amylase. 

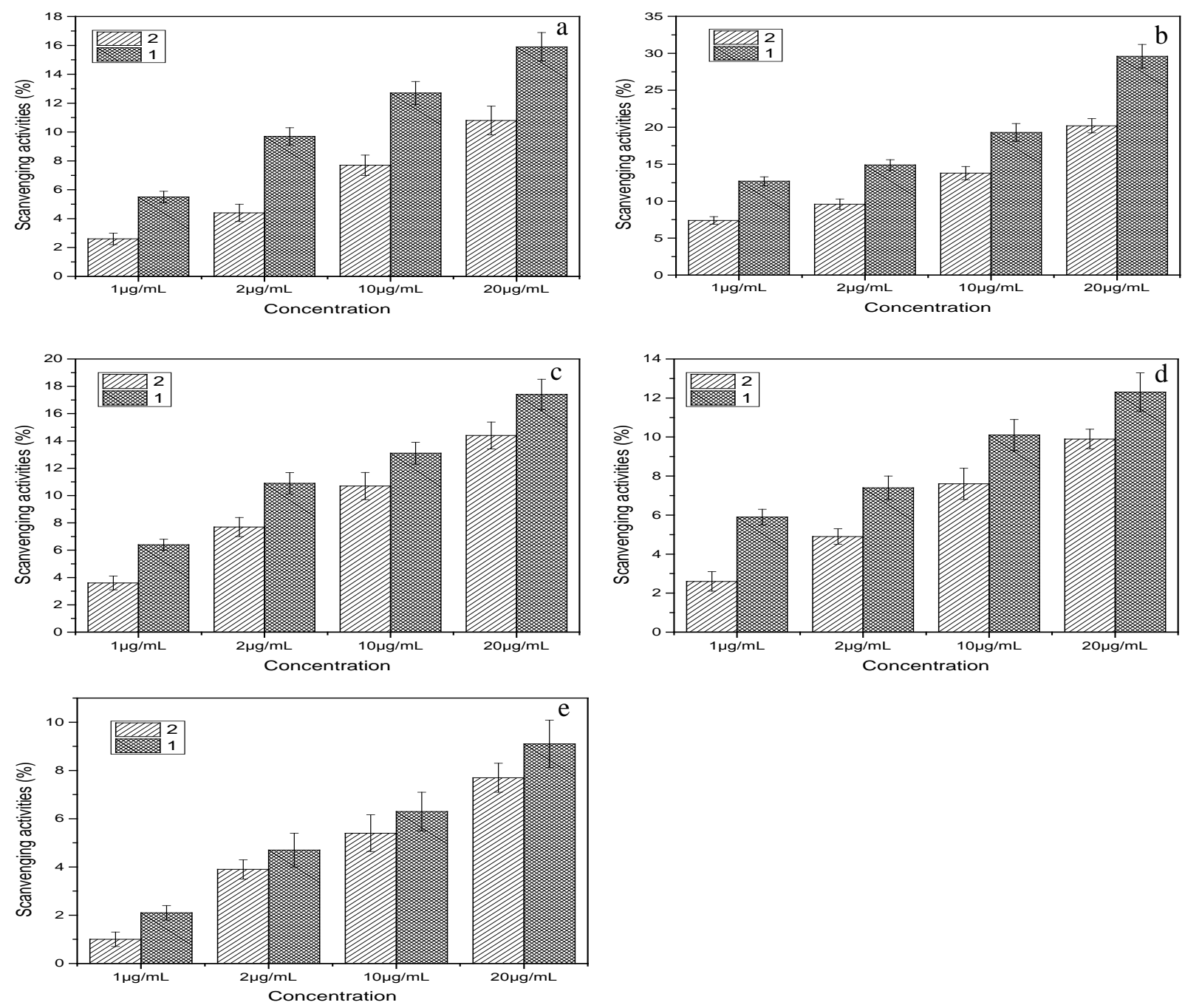

Fig.4 Effect of $\alpha$-amylase on the scanvenging activity of peanut shells polyhenols.

The histograms showed the antioxidant capacity of peanut shells polyhenol itself; the 2 histogram indicates the antioxidant capacity of peanut shells polyhenol after reacting with $\alpha$-amylase. $X$ position stretches as the concentration reduces. a, Effect of $\alpha$-amylase on the scanvenging activity of polyphenols extractions of peanut shells by ultrahigh pressure extraction. b, Effect of $\alpha$-amylase on the scanvenging activity of luteolin. c, Effect of $\alpha$-amylase on the scanvenging activity of phlorglucinol. d, Effect of $\alpha$-amylase on the scanvenging activity of pyrogallol. e, Effect of $\alpha$-amylase on the scanvenging activity of catechin.

This result could be explained from a structural point of view, this trend is corresponding with the complexity of different polyphenols. As known, peanut shells polyphenols are a natural compound of various kinds of polyphenols, it had the most complex structure. Phlorglucinol weighs 286.23 of a molecule, ranking second in complexity, showed secondary Rp in response. It could be concluded that the larger and complex polyphenols were easier to bind with $\alpha$-amylase, which leading to a more decrease in scavenging activities. This interpretation agrees with prior experiment in which researcher found that dissociation constants indicates that the larger and more complex polyphenols interact more strongly with proline-rich proteins in salivary. Moreover, phlorglucinol presents a carbonyl group $\mathrm{C}=\mathrm{O}$ in position $\mathrm{C}$ (4) indicating that this functional group could be important in the polyphenols' binding to $\alpha$-amylase. The importance of the presence of a carbonyl group at C (4) in the polyphenolic molecule has been reported. Diniz studied the characterization of interactions between polyphenolic compounds and human serum proteins by capillary electrophoresis, results indicated that a carbonyl group at $\mathrm{C}$ (4) in the 
polyphenolic molecule showed great effect on interaction between polyphenols and $\alpha$-amylase. Thus the absence of a carbonyl group at C (4) in their structure might explain why Rps of luteolin, pyrogallol and catechin were lower than that of phlorglucinol.

\section{Conclusions}

Antioxidative property of peanut shells polyphenols indicated that it could be used as a kind of potential natural antioxidant. But the results showed that the property of easier binding to proteins made its scanvenging activities decresed when in complex biological mixtures. The scanvenging activities of peanut shells polyphenols extracts, phlorglucinol, luteolin, pyrogallol, and catechin were all decreased when binding to the $\alpha$-amylase. Study also showed that, not only did polyphenols got restrained by $\alpha$-amylase secreted in the oral cavity and gut, polyphenols contents are also reduced by cooking methods like heating. So to make full use of its scavenging activity, we not only consider antioxidant capacity of a compound itself, but also its job circumstances.

\section{Acknowledgment}

This work is supported by the China Scholarship Council and the Science and Technology Department of Jilin Province (20140201018NY).

\section{References}

[1] Baxter, N. J., Williamson, M. P. Temperature dependence of chemical shifts in proteins. J. Biomol. NMR, 1997, 9, 359-369.

[2] Brunet, M.J., Blade, C., Salvado, M.J., Arola, L. Human apo A-I and rat transferring are the principal plasma proteins that bind wine catechins. J. Agric. Food. Chem., 2002, 24, 2708-2712.

[3] Serafini, M., Ghiselli, A., Ferro-Luzzi, A. In vitro antioxidant effect of green and black tea in man. Eur. J. Clin. Nutr., 1996, 50, 28-32.

[4] Arts, M. J. T. J., Dallinga, J. S., Voss, H. P., Haenen, G. R. M. M., Bast, A. Masking of antioxidant capacity by the interaction of flavonoids with protein. Food Chem. Toxicol., 2001, 39, 787-791.

[5] Arts, M. J. T. J., Dallinga, J. S., Voss, H. P., Haenen, G. R. M. M., Bast, A. A critical appraisal of the use of the troloxequivalent antioxidant capacity (TEAC) assay in defining optimal antioxidant structures. Food Chem., 2003, 80, 409-414.

[6] Papadopoulou A, Frazier R.A. Characterization of protein-polyphenol interactions. Trends. Food Sci. Tech., 2004, 15 (3-4), 186-190.

[7] Re, R., Pellegrini, N., Proteggente, A., Pannala, A., Yang, M., Rice-Evans, C. Antioxidant activity applying an improved ABTS radical cation decolorization assay. Free Radical Biol. Med., 1999, 26, 1231-1237.

[8] Berg, R.V.D., Haenen, G.R.M.M., Berg, H.V.D., Bast, A. Applicability of an improved Trolox equivalent antioxidant capacity (TEAC) assay for evaluation of antioxidant capacity measurements of mixtures. Food Chem., 1999, 66, 511-517. 